\title{
LA EVALUACIÓN DE LA ACTIVIDAD DOCENTE DE LA ESCUELA DE ENFERMERÍA DE LA UNIVERSIDAD DE COSTA RICA
}

\author{
Ernestina Aguirre Vidaurre ${ }^{1}$ \\ Docente de la Escuela de Enfermería y \\ Miembro del Consejo Universitario de la Universidad de Costa Rica \\ San José, Costa Rica
}

Recibido 9-VIII-2007 • Aceptado 27-VIII-2007

\begin{abstract}
Resumen: El presente artículo es un avance de una investigación cualitativa, en la cual se aplicó como método estructural la investigación fenomenológica, entre las técnicas utilizadas para la recolección de información están: la observación participante, la entrevista en profundidad y el grupo focal. La muestra fue de tipo intencional, participaron 3 exdirectoras y 1 directora, 25 docentes en Régimen Académico (propiedad), 23 docentes clínicos, 36 estudiantes de $V$ año (2002-2005-2006). La validación se llevó a cabo mediante la triangulación de las técnicas utilizadas. La motivación para investigar el tema, nació como una inquietud docente de explicar como se da la evaluación y buscar nuevas formas para evaluar la enseñanza y el aprendizaje en el quehacer de la enfermería en docencia. Por tanto, la propuesta fue elaborar los instrumentos, que se presentan.
\end{abstract}

Palabras clave: Evaluación, formularios, docencia, enfermería.

\section{Introducción}

Toda evaluación de la calidad de las universidades se centra en valorarla y medirla, considerando que la calidad de la producción de conocimientos depende solamente del docente. El tipo de conocimientos que se transmite, las personas que lo aceptan, las condiciones organizativas, administrativas y ambientales, así como la época y el contexto social y educativo, constituyen aspectos no tomados en cuenta para ponderar la calidad de la docencia, al ser la docencia la razón de la existencia de las universidades.

La gestión o el poder administrativo en la Universidad aprecia la docencia; es el centro de su preocupación, luego su evaluación constituye un aspecto prioritario en su quehacer, para enmendar, mejorar o para reproducir sus logros. Por ello se ha creado y se crean estructuras burocráticas para el control de los docentes: asistencia, confección y cumplimiento con los programas, horas asistencia a los estudiantes, controles horarios, etc., o en otros casos, complejas estructuras para evaluar el nivel académico de los docentes, en donde la 


\begin{abstract}
This article is an advance of a qualitative investigation in which, as a structural method, the phenomenological investigation was applied. Some of the techniques used for the information gathering process are: the participant observation, the in-depth interview, and the focus group. The sample was of intentional type. Three former chairpersons and one chairperson, 25 professors in "Regimen Académico" (steady position), 23 clinic professors, and 36 students of fifth year (2002-2005-2006) participated in this research. The validation was carried out by means of a triangulation of the techniques used. The motivation to investigate the subject arises as an educational inquiry to explain how evaluation occurs and find new ways of evaluating the teaching and learning processes in the daily work of infirmary teaching. Therefore, the proposal was to elaborate the instruments provided here.
\end{abstract}

Key words: Evaluation, forms, teaching, infirmary. formación, experiencia y producción constituyen aspectos sustantivos.

Por estas consideraciones la evaluación de los docentes o, mejor dicho los formatos para la "evaluación" son múltiples, e incluso variados en cada universidad, según las unidades académicas y las autoridades universitarias. El poder administrativo universitario evalúa a los docentes a partir de la apreciación de los estudiantes.

La docencia es evaluada por un poder que se ha autonomizado de la actividad académica. Los expertos en evaluación docente pueden evaluar sin conocer las peculiaridades de los maestros, de los estudiantes, de los contenidos que se imparten, de las condiciones y recursos de la docencia, etc. La pretendida evaluación objetiva y neutra nos muestra su precariedad y su gran compromiso con el poder y el status que solo encuentra a los docentes responsables y culpables del rendimiento del estudiante y de calidad de los estudios universitarios.

\section{Justificación}

La evaluación de la docencia, por obra de los expertos, ha dejado absurdamente de ser una labor académica para convertirse en una labor técnica, rígida y mecánica, que puede ser regulada y controlada administrativamente, sin el auxilio ni la participación de los académicos evaluados. La administración burocratizada decide cómo ponderar los trabajos e incluso, a quién renovar o suspender las labores docentes. Los procesos administrativos universitarios han subordinado a la academia y la docencia. Una administración que no está al servicio de la docencia, que se ha independizado y supeditado a lo administrado, es una inversión que niega la propia concepción de administración. Según Chiavenato (2000) "La administración no es un fin en sí misma, pero sí un medio de lograr que las cosas se realicen de la mejor manera posible, al menor costo y con la mayor eficiencia y eficacia”. (p. 9) 
Nadie puede negar que la evaluación sea una actividad compleja pero constituye una tarea necesaria y fundamental en la labor docente.

En las prácticas de evaluación puede incluirse lo relativo a procedimientos, técnicos, instrumento y criterios. Los instrumentos empleados en la Universidad y, específicamente en la Escuela de Enfermería, no evalúan las capacidades del docente porque se evalúa únicamente la parte teórica y no la práctica. Además, esta evaluación la realizan solamente los estudiantes y las estudiantes, incumpliendo lo acordado en el Centro de Evaluación Académica y violentándose, tanto el principio de la evaluación, como los lineamientos para la implementación de un Modelo de Gestión de la Calidad en la Universidad de Costa Rica,

(....) el reconocimiento se encuentra ligado al papel que desempeñan las personas en las organizaciones. Si hay una implicación positiva del personal, ésta se ve correspondida con un reconocimiento explícito de ese esfuerzo. La organización se legitima ante su personal si lo hace partícipe de los progresos obtenidos. (Consejo Universitario, 2004, p. 1)

Estos instrumentos están orientados a otros fines como:

- Ascenso en Régimen Académico.

- Evaluar los contenidos aprendidos por los alumnos del nuevo plan de estudio.

- Actividades de enseñanza y gestión realizada por el propio docente.

\section{Objetivo general}

- Conocer el proceso de evaluación aplicado a las docentes de la Escuela de Enfermería.

\section{Objetivos específicos}

- Analizar el proceso de evaluación utilizado en la unidad Académica.
- Analizar los métodos aplicados por la Directora de la Unidad Académica para evaluar a las docentes.

- Analizar las razones y los efectos de esa evaluación en las docentes.

\section{Marco teórico}

La evaluación de la enseñanza debe entenderse como una reflexión constante y necesaria y considerarse como parte de la formación docente y no como se tiene establecido en la Universidad, específicamente en la Escuela de Enfermería, que la podemos catalogar como oculta, pues no se evalúa en la práctica. Los informantes claves no solo mencionan lo positivo, también refieren las debilidades encontradas; sin embargo, esas debilidades no se corrigen, pues se anotan en las evaluaciones y nunca se les comunica a las profesoras.

Según Chiavenato (2002) la evaluación del desempeño debe proporcionar beneficios a la organización y a las personas; en consecuencia, se debe tener en cuenta las siguientes líneas básicas:

- La evaluación debe abarcar no sólo el desempeño en el cargo ocupado, sino también, el alcance de las metas y los objetivos. Desempeño y objetivos deben ser temas inseparables de la evaluación del desempeño.

- La evaluación debe hacer énfasis en el individuo que ocupa el cargo y no en la impresión respecto a los hábitos personales observados en el trabajo. La evaluación se debe concentrar en un análisis objetivo del desempeño y no en la apreciación subjetiva de hábitos personales. Empeño y desempeño son cosas distintas.

- La evaluación debe ser aceptada por ambas partes: evaluador y evaluado. Ambos deben estar de acuerdo en que la evaluación debe traer algún beneficio benéfico para la organización y para el empleado. 
- La evaluación del desempeño se debe utilizar para mejorar la productividad del individuo en la organización, equipándolo mejor para producir con eficacia y eficiencia.

Es importante que la director(a) y los coordinadores(as) asuman la cultura de la evaluación del desempeño, el manejo de la técnica, su trascendencia y sus efectos. Porque quien debe evaluar a la docente o el docente es la directora o la coordinadora, porque son las que deben hacer un verdadero seguimiento, con un diagnóstico de los puntos fuertes y débiles de la docente.

\section{Resultados}

\section{Sujetos y métodos}

Se aplicó como método estructural la investigación fenomenológica. Entre las técnicas utilizadas para la recolección de información están: la observación participante, la entrevista en profundidad y el grupo focal.

La muestra fue de tipo intencional, participaron 3 exdirectoras y 1 directora, 25 docentes en Régimen Académico (en propiedad), 23 docentes clínicos, 36 estudiantes de V año (2002-2005-2006).

La validación se llevó a cabo mediante la triangulación de las técnicas utilizadas.

Tabla 1

Escuela de Enfermería. Opinión de los docentes en régimen sobre la evaluación del docente Sede Rodrigo Facio, Noviembre 2002, 2005, 2006

\begin{tabular}{|c|c|c|c|c|c|c|}
\hline \multirow{4}{*}{ AFIRMACIONES } & \multicolumn{6}{|c|}{ AÑOS } \\
\hline & \multicolumn{2}{|c|}{2002} & \multicolumn{2}{|c|}{2005} & \multicolumn{2}{|c|}{2006} \\
\hline & \multicolumn{6}{|c|}{ ESCALA } \\
\hline & $\mathrm{Si}$ & No & $\mathrm{Si}$ & No & $\mathrm{Si}$ & No \\
\hline Es la única evaluación que existe. & 25 & 0 & 25 & 0 & 25 & 0 \\
\hline $\begin{array}{l}\text { Yo tengo años de laborar en la escuela y solo me han evaluado cuando he } \\
\text { solicitado ascenso en régimen académico. }\end{array}$ & 15 & 10 & 15 & 10 & 20 & 5 \\
\hline $\begin{array}{l}\text { Yo como tengo pocos años de laborar en la escuela y no he solicitado } \\
\text { ascenso en régimen académico no me han evaluado. }\end{array}$ & 7 & 15 & 7 & 15 & 7 & 15 \\
\hline $\begin{array}{l}\text { Yo cuando fui Directora de la Escuela de Enfermería realicé evaluaciones } \\
\text { únicamente cuando las solicitaba el Centro de Evaluación Académica } \\
\text { para ascenso en régimen de una docente. }\end{array}$ & 3 & 0 & 3 & 0 & - & - \\
\hline En la Universidad no existe un sistema de evaluación permanente. & 1 & & 1 & & 1 & \\
\hline
\end{tabular}

Fuente: Entrevista en profundidad docentes en régimen.

Otro dato importante, la evaluación es únicamente para las docentes en Régimen, las docentes clínicas no pueden participar porque la normativa las excluye, como se observa en el siguiente cuadro. 
Cuadro 1

Escuela de Enfermería la evaluación según docentes clínicos Sede Rodrigo Facio. Noviembre 2002, 2005, 2006.

\begin{tabular}{cccc}
\hline \multirow{2}{*}{ Evaluación } & \multicolumn{3}{c}{ AÑOS } \\
\cline { 2 - 4 } & 2002 & 2005 & 2006 \\
\hline Si & 0 & FRECUENCIA & 0 \\
No & 23 & 25 & 23 \\
\hline
\end{tabular}

Fuente: Entrevista docentes clínicos.

Lo que mencionan los estudiantes.

Tabla 2

Escuela de Enfermería. Opinión de los estudiantes de V Año de Licenciatura de Enfermería sobre la evaluación docente de la práctica clínica. Periodo 2002-2005-2006 Sede Rodrigo Facio, noviembre 2006

\begin{tabular}{|c|c|c|c|c|c|c|}
\hline \multirow{4}{*}{ AFIRMACIONES } & \multicolumn{6}{|c|}{ AÑOS } \\
\hline & \multirow{2}{*}{\multicolumn{2}{|c|}{2004}} & \multirow{2}{*}{\multicolumn{2}{|c|}{$\frac{2005}{\text { ESCALA }}$}} & \multicolumn{2}{|c|}{2006} \\
\hline & & & & & & \\
\hline & $\mathrm{Si}$ & No & $\mathrm{Si}$ & No & $\mathrm{Si}$ & No \\
\hline Compromiso de parte de la docente en asumir su rol. & 3 & 12 & 3 & 12 & 3 & 12 \\
\hline $\begin{array}{l}\text { Hay incongruencia, en algunas profesoras entre el plan de estudios y los } \\
\text { ejes temáticos con lo que enseñan y la práctica. }\end{array}$ & 11 & 1 & 11 & 1 & 11 & 1 \\
\hline $\begin{array}{l}\text { Integran los ejes temáticos en la práctica ejemplo ética, derechos huma- } \\
\text { nos, gerencia en enfermería y género. }\end{array}$ & 4 & 8 & 4 & 8 & 4 & 8 \\
\hline $\begin{array}{l}\text { Se van al extremo de que el estudiante o la estudiante decida cómo hacer } \\
\text { las cosas o le dicen cómo hacer todo. }\end{array}$ & 4 & 8 & 4 & 8 & 4 & 8 \\
\hline No funcionan como facilitadoras. No los orientan adecuadamente. & 3 & 9 & 0 & 12 & 3 & 12 \\
\hline $\begin{array}{l}\text { Ejemplifican tomando a niños o niñas en el Hospital, a los cuales no hay } \\
\text { no se les aplica nada. }\end{array}$ & 11 & 1 & 11 & 1 & 11 & 1 \\
\hline \multicolumn{7}{|l|}{$\begin{array}{l}\text { No hay integración de la teoría con la práctica como la administración de } \\
\text { medicamentos, nutrición y las teorizantes. }\end{array}$} \\
\hline Se continúa muy arraigado al paradigma de la categorización. & 3 & 12 & 3 & 12 & 3 & 12 \\
\hline No hay una discusión del hacer, del ser, del saber de Enfermería. & 12 & 0 & 12 & 0 & 12 & 0 \\
\hline No cumplen con el horario establecido. & 4 & 8 & 4 & 8 & 4 & 8 \\
\hline $\begin{array}{l}\text { Profesoras no las deja realizar procedimientos por temor y esa inseguri- } \\
\text { dad se los trasmite a los alumnos y las alumnas. }\end{array}$ & & & & & & \\
\hline
\end{tabular}

Fuente: Población estudiantil que participó en la investigación como grupo focal.

\section{Discusión}

\section{Compromiso de parte de la docente en asumir su rol}

Hay incongruencia en algunas profesoras entre el discurso de lo que es el plan de estudios y los ejes temáticos, y lo que enseñan y hacen en la práctica. Integran los ejes temáticos en la práctica, por ejemplo ética, derechos humanos, gerencia en enfermería y género. Se van al extremo de que el estudiante o la estudiante decida cómo hacer las cosas o le dicen como hacer 
todo. No funcionan como facilitadoras o los dejan solos o no los dejan ni un momento solas(os).

Deben tener criterios formales e informales para valorar la competencia del docente:

- Comprobar el proceso y autocontrol, y conocer si conduce al alumno en el sentido correcto.

- Valorar cuantitativa y cualitativamente el tipo de apoyo prestado a los estudiantes.

- Esta evaluación le proporcionará al docente información importante sobre su quehacer; aspectos por mejorar y reforzar en lo didáctico, recursos pedagógicos.

La vigilancia permanente de las actividades realizadas en la enseñanza le permite al docente contar con bases suficientes para el logro de la continuidad necesaria durante el curso o secuencia educativa y también para establecer y mantener contextos indispensables.

\section{Consideraciones finales}

- La evaluación de la práctica docente, como regularmente se efectúa en la Universidad, deberá ser redefinida para permitir una adecuación permanente en la interacción entre el docente, el estudiante y las poblaciones atendidas y capacitadas.

- La práctica de la enseñanza de la enfermería, procura hacer frente a las nuevas exigencias de los servicios de salud, así como mejorar la calidad de atención, lo cual potencializa el papel de la Enfermería a los futuros profesionales.

- $\quad$ El ejercicio de la formación en enfermería es complejo; abarca un amplio espectro de actividades en torno de la salud, en las competencias y dominio de las técnicas de la formación práctica y de la investigación. Estas condiciones son requisitos ante una realidad laboral caracterizada por ser tensa e intensa, ante circunstancias que han afectado las políticas públicas en salud con escasez de personal y en condiciones no adecuadas de trabajo.

Muchas de las causas de la insatisfacción de la labor de las docentes clínicas, podrían evitarse si los procesos administrativos respondieran de manera directa a las peculiaridades de la labor académica y no por prácticas mecánicas y estandarizadas. Asimismo, es posible paliar los inconvenientes respetando el derecho de petición de la Unidad Académica, en cuanto a mejorar el salario a contratar a las docentes clínicas con un mes de anticipación y a permitir que la docente en Enfermería que labora en otra institución pública tenga derecho a la sobreposicion horaria, como ocurre con otros profesionales del Área de Salud que laboran para la Universidad de Costa Rica.

- Con los resultados obtenidos de las entrevistas, así como de las respuestas del grupo focal (docentes clínicasestudiantes), se puede inferir que son las profesoras en propiedad y de tiempo completo de la Escuela, las que tienen una mejor formación académica y dedicación y no las docentes clínicas. El conocimiento de esta situación trasciende el nivel decisorio de la Escuela y compromete a la Universidad en su normatividad y tradición.

- En las fallas o debilidades como docentes todos coinciden: son excelentes enfermeras, pero se les dificulta la enseñanza. Al comparar estos resultados con la situación encontrada en las docentes en Régimen, se logra inducir que corresponden a la falta de educación continua sobre didáctica, 
pedagogía, enseñanza; únicamente se hace un curso de didáctica como obligación para ingresar a Régimen Académico. Cuando se contratan es sin experiencia en docencia.

- Los instrumentos que se emplean en la Universidad y específicamente en la Escuela de Enfermería, no evalúan las capacidades como docente porque se evalúa únicamente la parte teórica y no la práctica.

Además, esta evaluación la realizan solamente los estudiantes y las estudiantes, incumpliendo lo acordado en el Centro de Evaluación Académica y violentándose, tanto el principio de la evaluación, como los lineamientos para la implementación de un Modelo de Gestión de la Calidad en la Universidad de Costa Rica.

“.... el reconocimiento se encuentra ligado al papel que desempeñan las personas en las organizaciones. Si hay una implicación positiva del personal, ésta se ve correspondida con un reconocimiento explícito de ese esfuerzo. La organización se legitima ante su personal si lo hace partícipe de los progresos obtenidos." (Consejo Universitario, 2004: 1)

\section{- Propuestas de dos instrumentos}

Si se considera esta investigación, la búsqueda de un cambio práctico para efectuar la evaluación del recurso humano docente de la Escuela de Enfermería debe trascender del obligatorio, publicar resultados y transitar de la forma como se evalúa actualmente, hacia la evaluación deseada. Por tanto, era un reto elaborar instrumentos, que permitan lograr ese cambio, los instrumentos que se elaboraron se presentan en el anexo \#1 y \#2.

\section{Nota}

1. Docente de la Escuela de Enfermería y Miembro del Consejo Universitario de la Universidad de Costa Rica. ernesagui@hotmail.com

\section{Referencias bibliográficas}

Álvarez, J. L. (2003). Cómo hacer investigación cualitativa: Fundamentos y Metodología. México D. F.: Editorial Piado Mexicana S. A.

Armstrong, M. (1991). Gerencia de recursos humanos: integrado el personal y la empresa. Bogotá: Fondo Editorial Legis.

Consejo Universitario. (2004). Lineamientos para la implementación de un Modelo de Gestión de la Calidad en la Universidad de Costa Rica. San José: Universidad de Costa Rica. Publicados en Gaceta Universitaria.

Chiavenato, I. (1990). Administración de recursos humanos. México. D. F.: Editorial McGraw-Hill Interamericana.

Chiavenato, I. (2000). Introducción a la Teoría General de la Administración. Bogotá: Editorial McGraw-Hill Interamericana, S. A.

Daria, K. y Newstrom, J. (1991). El comportamiento humano en el trabajo: comportamiento organizacional. México. D. F.: Editorial McGraw-Hill Interamericana.

De Alba, A. (1997). El currículo universitario. México. D. F.: Editores Plaza y Valdés.

Flores, R. (1994). Hacia una pedagogía del conocimiento. Bogotá: McGraw-Hill.

Mackerman, J. (1999). Investigación. Acción y currículo. Madrid: Ediciones Mareta.

Huaylupo, J. (1994). La evaluación del desempeño ¿un enfoque individual del trabajo colectivo o una visión individualista del trabajo social? En: 
Revista de Servicio Civil, 2 (EneroJunio), pp. 21-36.

Mora, Benjamín y Solís, Vivianne. (1989). El quehacer académico de las Universidades Estatales de Costa Rica en relación al medio ambien- te. En: Revista Estudios Sociales Centroamericanos, 49, (Enero-Abril), pp. 13, 24.

Mody R., Robert, N. (1997). Administración de Recursos Humanos. México: Editorial Pearson Educación. 


\section{ANEXOS \\ UNIVERSIDAD DE COSTA RICA \\ SISTEMA DE ESTUDIOS DE POSGRADO \\ MAESTRÍA EN ADMINISTRACIÓN PÚBLICA \\ EVALUACIÓN DE LA PERSONA DOCENTE \\ REALIZADA POR COORDINADORA}

Módulo:

Lugar:

Docente:

Categoría: Docente Régimen:

Clínico sin experiencia Egresada Escuela

\begin{tabular}{|c|c|c|c|c|}
\hline ASPECTOS & SI & NO & No Aplica & OBSERVACIONES \\
\hline \multicolumn{5}{|l|}{$\begin{array}{l}\text { El programa del módulo por } \\
\text { parte del docente fue: }\end{array}$} \\
\hline \multicolumn{5}{|l|}{ Entregado al inicio del ciclo } \\
\hline \multicolumn{5}{|l|}{ Discutido al inicio } \\
\hline \multicolumn{5}{|l|}{ Entregado y discutido al inicio } \\
\hline \multicolumn{5}{|l|}{ No fue discutido } \\
\hline \multicolumn{5}{|l|}{ Discutido y entregado por otra u otro } \\
\hline \multicolumn{5}{|l|}{ La docente o el docente tiene: } \\
\hline \multicolumn{5}{|l|}{ Conocimiento de la materia } \\
\hline \multicolumn{5}{|c|}{ Calidad en el desarrollo de los contenidos } \\
\hline \multicolumn{5}{|c|}{ Claridad para explicar } \\
\hline \multicolumn{5}{|l|}{ Prepara clases } \\
\hline \multicolumn{5}{|l|}{ Asiste a dar lecciones } \\
\hline \multicolumn{5}{|c|}{$\begin{array}{l}\text { Realizó una evaluación prediagnóstica de la } \\
\text { estudiante o el estudiante }\end{array}$} \\
\hline \multicolumn{5}{|l|}{ Proporciona información actualizada } \\
\hline \multicolumn{5}{|c|}{$\begin{array}{l}\text { Las técnicas que utiliza para enseñar } \\
\text { fueron: }\end{array}$} \\
\hline \multicolumn{5}{|c|}{ Los gestos } \\
\hline \multicolumn{5}{|l|}{ Las preguntas y respuestas } \\
\hline \multicolumn{5}{|l|}{ El entusiasmo y motivación } \\
\hline \multicolumn{5}{|l|}{ Las metáforas } \\
\hline \multicolumn{5}{|l|}{ Los mapas conceptuales } \\
\hline \multicolumn{5}{|l|}{ Los gráficos } \\
\hline \multicolumn{5}{|l|}{ Las discusiones } \\
\hline \multicolumn{5}{|l|}{ La asignación de tareas o proyectos. } \\
\hline \multicolumn{5}{|l|}{ La persona docente } \\
\hline \multicolumn{5}{|c|}{$\begin{array}{l}\text { Estimula la capacidad de razonamiento en el } \\
\text { estudiante }\end{array}$} \\
\hline Promueve la curiosidad intelectual & & & & \\
\hline
\end{tabular}


continuación...

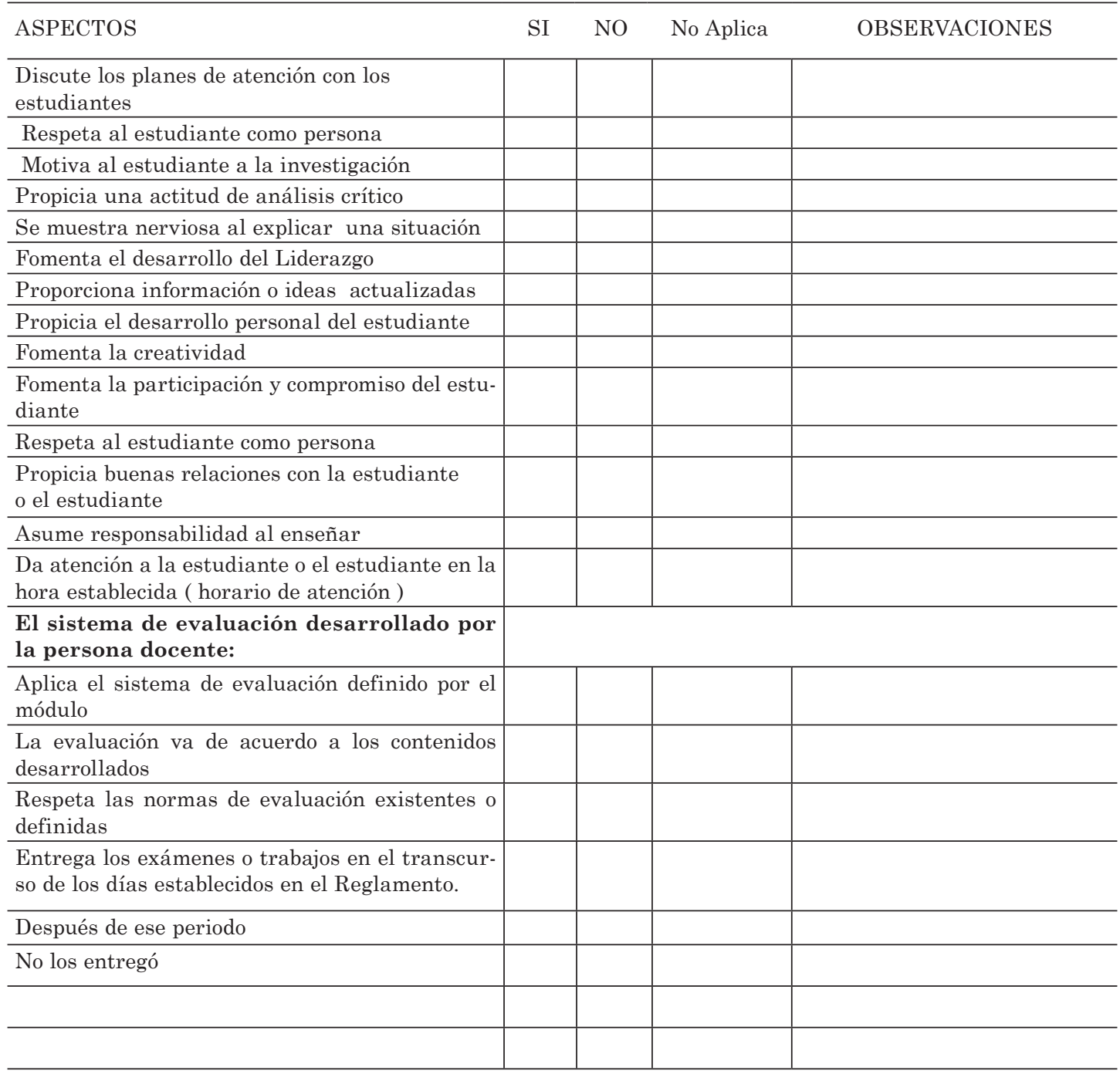

Elaborado por Ernestina Aguirre Vidaurre. 


\section{UNIVERSIDAD DE COSTA RICA ESCUELA DE ENFERMERÍA \\ EVALUACIÓN DE LA PERSONA DOCENTE REALIZADA POR ESTUDIANTES}

Nombre del curso-taller-módulo:

Docente:

Fecha:

Período:

I PARTE. Percepción del desarrollo del curso. Marque con una X la casilla que mejor se ajuste a su criterio.

\begin{tabular}{|c|c|c|c|c|}
\hline ESCTRUCTURA CURRICULAR & $\begin{array}{l}\text { Excelente } \\
\text { (4) }\end{array}$ & $\begin{array}{c}\text { Muy } \\
\text { Bueno(3) }\end{array}$ & $\begin{array}{l}\text { Bueno } \\
(2)\end{array}$ & $\begin{array}{l}\text { Deficiente } \\
\text { (1) }\end{array}$ \\
\hline \multicolumn{5}{|l|}{ 1. Se discutió el programa de manera: } \\
\hline \multicolumn{5}{|l|}{ 2. El programa se ejecutó de acuerdo a lo planeado: } \\
\hline \multicolumn{5}{|l|}{$\begin{array}{l}\text { 3. El programa respondió a sus expectativas } \\
\text { en forma: }\end{array}$} \\
\hline \multicolumn{5}{|l|}{$\begin{array}{l}\text { Cómo califica la ejecución del curso en una escala de } \\
0 \text { a } 100\end{array}$} \\
\hline \multicolumn{5}{|l|}{$\begin{array}{l}\text { DESEMPEÑO DOCENTE: } \\
\text { 1. El dominio del tema por parte del docente ha sido: }\end{array}$} \\
\hline \multicolumn{5}{|l|}{$\begin{array}{l}\text { 2. Manejo del material didáctico y de los recursos } \\
\text { audiovisuales se ha hecho de manera: }\end{array}$} \\
\hline \multicolumn{5}{|l|}{ 3. El uso del tiempo ha sido: } \\
\hline \multicolumn{5}{|l|}{$\begin{array}{l}\text { Cómo califica el desempeño docente en una escala } \\
\text { de } 0 \text { a } 10 \square \\
\text { Justifique su respuesta: }\end{array}$} \\
\hline \multicolumn{5}{|l|}{$\begin{array}{l}\text { MATERIAL DIDÁCTICO Y RECURSOS } \\
\text { AUDIOVISUALES: } \\
\text { 1. Contiene las ideas centrales de la temática estudia- } \\
\text { da de manera: }\end{array}$} \\
\hline \multicolumn{5}{|l|}{ 2. Estimula el aprendizaje de manera: } \\
\hline \multicolumn{5}{|l|}{$\begin{array}{l}\text { 3. La variedad en el uso de recursos audiovisuales } \\
\text { ha sido: }\end{array}$} \\
\hline \multicolumn{5}{|l|}{$\begin{array}{l}\text { INFRAESTRUCTURA / MOBILIARIO: } \\
\text { 1. La ventilación en el espacio físico utilizado es: }\end{array}$} \\
\hline 2. La iluminación en el espacio físico e higien es: & & & & \\
\hline
\end{tabular}

Elaborado por Ernestina Aguirre Vidaurre. 
II. PARTE. Anote de manera puntual las preguntas planteadas a continuación:

1. Anote los aspectos positivos del (Curso-taller-modulo)

2. Anote los aspectos que deben mejorar en el desarrollo del (Curso-tallermódulo)

3. ¿Que opinión le merece el sistema de evaluación utilizado?

4. ¿Qué opinión le merece la metodología empleada durante el desarrollo del (Curso-taller-módulo)?

5. Describa las principales facilidades y limitaciones que se presentaron en cuanto al desempeño de la docente en el desarrollo del (Curso-taller-módulo)

\begin{tabular}{l|l|l}
\hline \multicolumn{1}{c|}{ DOCENTE } & FACILIDADES & LIMITACIONES \\
\hline $\begin{array}{l}\text { Coordinadora } \\
\text { (Curso-taller-módulo): }\end{array}$ & & \\
& & \\
\hline Docente Teórica: & & \\
& & \\
\hline Docente Práctica: & & \\
& & \\
\hline
\end{tabular}

